\title{
Drug bioavailability from topically applied ocular drops. Does drop size matter?
}

\author{
Anselm G.M. Jünemannn ${ }^{1,2}$, Tomasz Chorągiewicz², Małgorzata Ozimek2, Paweł Grieb', Robert Rejdak²,3 \\ 'Department of Ophthalmology, University Eye Hospital, Rostock, Germany \\ ${ }^{2}$ Department of General Ophthalmology, Medical University of Lublin, Lublin, Poland \\ ${ }^{3}$ Department of Experimental Pharmacology, Mossakowski Medical Research Centre, Polish Academy of Sciences, Warsaw, Poland
}

\begin{abstract}
The application of drops containing ocular medicines to the conjunctival sac is the most common method of drug delivery to the anterior segment of the eye. Although this route of application seemingly displays numerous advantages, obtaining effective drug concentration at its site of action is challenging. The bioavailability of a topically applied drug depends on various factors related to the eye, to the drug and formulation, to the drop, and to the patient. The present article discusses their relative significance. From a drop applied to an eye, at most $5 \%$ of a drug dose enters the ocular structures. Of utmost importance for effective ocular drug delivery are patient compliance and the physicochemical properties of the drug. For a given concentration of an active substance, drop size may determine drug adverse effects but does not influence its efficacy.
\end{abstract}

KEY WORDS: ocular drops, drug bioavailability, side effects, patient compliance

Ophthalmol J 2016; Vol. 1, No. 1, 29-35

\section{INTRODUCTION}

Application of drops containing a medicine topically to the conjunctival sac is the most common method of ocular drug delivery. Such applied medicines are widely used for the treatment of diseases of the anterior segment of the eye: cornea, conjunctiva, episclera, and sclera, as well as deeper intraocular structures: iris and ciliary body. Antibiotics, steroids, non-steroid anti-inflammatory drugs, and IOP-decreasing anti-glaucoma drugs are given in such a way. The advantages of eye drops are frequently presented to include the following: relative ease and non-invasiveness of self-administration, direct targeting of the desired destination, bypassing the first-pass liver metabolism, and minimising systemic side effects. In reality, for an ocular drug topically applied, such as eye drops, reaching an effective concentration at the site of action and maintaining it for a desired time is challenging, mainly because of the short ocular contact time of the active principle due to it being washed away through the lacrimal system, diluting with tears, and poor penetration to the eye.

To reach a destination site, a drug applied in eye drops must cross anatomical barriers and bypass protective mechanisms of the eye. When we consider so-called innovator drugs that receive market authorization on the basis of a full registration dossier that includes clinical studies proving safety and therapeutic efficacy, particulars of drug substance penetration into, and retention at, the site of action are of no practical importance. The situation is different when we consider generics.

The European Medicines Agency [1] defines generic medicine as a medicine that is developed to be the same as a medicine that has already been authorised (the 'reference medicine'). Furthermore, it is stated that a generic medicine contains the same quantity of active substance(s) as the reference medicine. However: The inactive ingredients, or 'excipients', may 
differ between the generic medicine and its reference. [This is possible because] ...the active substance of a medicine is what gives it its therapeutic effect.

Any pharmacological treatment requires delivery of an active substance to, and establishing its effective concentration at, a destination site for a required period of time. When a drug is given parenterally or orally, these conditions are in most cases approximated by the area under the curve (AUC), a parameter obtained by integrating drug concentrations measured in blood plasma at certain discrete time points. A generic variant of a reference drug is deemed acceptable if its average AUC value lies within an $80-125 \%$ interval from the average AUC of the reference drug and the difference is not statistically significant. However, in the case of eye drops the blood plasma AUC is irrelevant.

Multiple generic variants of reference formulations of eye drops are market approved in the European Union and the USA, but their comparability and interchangeability is sometimes debated, see e.g. [2]. Doubts are fuelled by reports on seemingly profound differences between various brands of eye drops. Apparently excipients and containers from which drops are delivered may markedly influence drug bioavailability; excipients can also alter tolerability to eye drops. For chronically applied drugs, such as those used to treat glaucoma, even small differences between drug brands may be of significance.

Mammo et al. [3] compared volumes of different brands of reference and generic topical eye drops available in the USA and Canada and found, inter alia, that one generic brand of timolol maleate (gel-based formulation) delivered approximately $40 \%$ less drop volume than its reference formulation, whereas the other generic brand of timolol maleate (ophthalmic solution formulation) delivered approximately $20 \%$ less drop volume than its reference formulation. Are these generics equivalent to their respective references? Leitritz et al. [4] compared the reference and 23 different generic brands of a leading anti-glaucoma drug latanoprost available in Germany and also found noticeable differences in the contents of both the active substance and the main excipient (benzalkonium chloride) in the drop's mass and the $\mathrm{pH}$ of the solution, and in bottle forms. The authors suggested that before prescribing generic eye drops in ophthalmology various factors have to be considered that might influence the therapeutic effect. Unfortunately, they did not provide any guidance on which factors are more, and which are less important.
The aim of the present article is to discuss factors that determine the bioavailability of active principles from topically applied eye drops. They are conventionally classified into four groups: eye-related, drug and formulation-related, drop-related, and patient-related. Although these factors influence each other, their relative importance, by which we mean their contribution to the overall performance of a drug, differ.

\section{EYE-RELATED FACTORS}

The human eye is an extremely important and sensitive sensory organ; at the same time, it is a part of the body surface in continuous contact with the outer environment, tightly protected from foreign bodies and substances with several anatomical barriers and precorneal factors. These protective mechanisms do not support bioavailability of drugs from eye drops.

Anatomical barriers for a topical route of drug administration consist of cornea, conjunctiva, and sclera. Each of these has a different structure, reflected by different permeability to drug substances [5]. The cornea and conjunctiva make the ocular surface. They are both covered with epithelium; additionally, mucous covers most of the outer surface of the eyeball [6]. The total surface area for the adult conjunctival sac of one eye (including the cornea) averages $16 \mathrm{~cm}^{2}$ [7], and the corneal surface is 17-times smaller than the conjunctival surface [8].

The cornea is avascular and consists of three layers: the epithelium, stroma, and endothelium. The corneal epithelium contains $90 \%$ of the total count of corneal cells, but it only represents ca. $10 \%$ of its thickness. It consists of six layers of non-keratinised stratified squamous epithelium, permeable to lipophilic agents but significantly resistant to hydrophilic agents. Intercellular permeability is additionally reduced by desmosomes and tight junctional complexes (zonulae occludens). Conversely, the corneal stroma, which represents $90 \%$ of the corneal thickness, is built up mostly of collagen with a small number of cells. The highly hydrated structure of the stroma is permeable to hydrophilic agents but constitutes a barrier to lipophilic molecules. The innermost layer of the cornea consists of a single layer of hexagonally-shaped endothelial cells. Junctions between these cells are leaky, enabling passage of molecules; therefore, the inner corneal epithelium is not considered a barrier for topically administered drugs. 
Compared to the cornea, the conjunctiva is less permeable for lipophilic agents. Furthermore, tight junctions of conjunctival epithelium also reduce its permeability to hydrophilic molecules [9]. Nonetheless, dense vascularisation with blood and lymphatic vessels leads to absorption of drugs into circulation and reduces their bioavailability at the destination site. Both conjunctiva and the lacrimal drainage system are covered with stratified columnar epithelium, whereas the nasal mucosa is covered with pseudostratified columnar epithelium with very highly vascularised lamina propria. Systemic absorption from nasal mucosa is much larger than from conjunctiva [10].

The other barrier on the route of a topically administered drug is the sclera, which is localised under the conjunctiva (known also as the white of the eye). Anatomically this tissue is continuous to the corneal stroma. It consists mostly of collagen fibres and proteoglycans. The permeability of drug molecules across the sclera is comparable to corneal stroma and is dependent on molecular radius and weight, hydrophilicity, and charge of the molecule. For hydrophilic agents it is 80 -times faster than through all corneal layers. It is inversely proportional to the molecular radius [11]. Positively charged molecules exhibit poor permeability, presumably due to their binding to the negatively charged proteoglycan matrix [12].

In summary, respective anatomical eye barriers are differently permeable to lipophilic and hydrophilic substances, to low- and high-molecular weight molecules, etc. Depending on physicochemical properties, drugs applied topically follow different routes across anatomical barriers to enter the anterior chamber, as shown in Figure 1.

In addition to the aforementioned anatomical barriers, the eye is guarded by precorneal factors, which include blinking, induced lacrimation, tear film turnover, and drainage. The tear film is a first line hindrance against pathogens. Because of its pivotal role for vision, refraction, and lubrication, it is described as a sixth layer of the cornea. It is about $8 \mu \mathrm{m}$ thick with a volume of $7 \mu \mathrm{L}$. The fluid from conjunctival sac is vacuumed to the lacrimal drainage system, lacrimal sac, and nasal cavity by a valve-like mechanism, described by Doane [14].

Tear production in normal conditions is about $1 \mu \mathrm{L} / \mathrm{min}$ with a fluid turnover rate of 0.5 to $2.2 \mu \mathrm{L} / \mathrm{min}$ [15]. Mechanical manipulation of eyelids needed to instil the drug will double or triple normal basal tear flow. Restoration of the normal

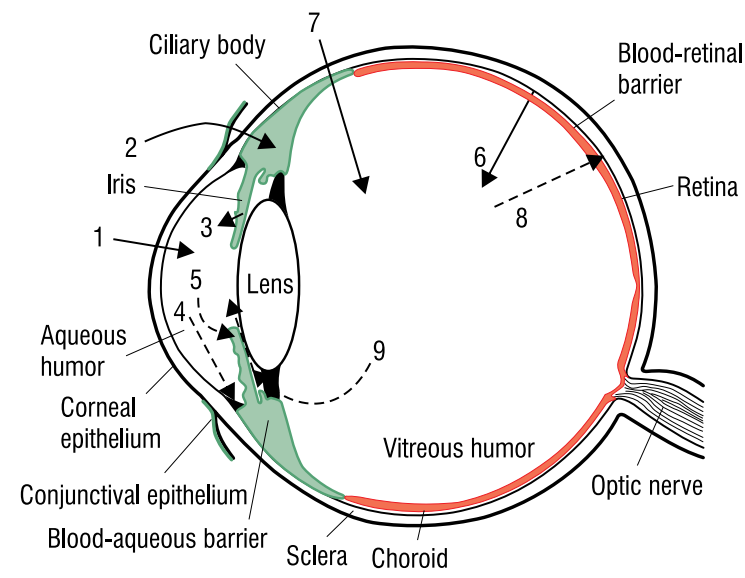

FIGURE 1. Pathways employed by drugs applied topically to cross anatomical barriers of the eye and enter the anterior chamber. The main route is assumed to be via the cornea (1). Large and hydrophilic drugs prefer the conjunctival and scleral route (2) and then diffuse into the ciliary body (3). From the anterior chamber the drugs are removed either by aqueous humour outflow (4) or by venous blood flow after diffusing across the iris surface (5). After systemic administration drugs must pass across the retinal pigment epithelium or the retinal capillary endothelium to reach the retina and vitreous humour (6). Alternatively, drugs can be administered by intravitreal injection (7). Drugs are eliminated from the vitreous via the blood-retinal barrier (8) or via diffusion into the anterior chamber (9). Reproduced from [13]

tear volume after application of the eye drop requires about two to three minutes, and most of the topically administered solution is drained from the conjunctival sac during the first $15-30$ seconds. The larger the volume instilled, the more rapidly it is drained through the nasolacrimal duct system [16]. The lacrimal system removes the agents 100-times faster than the cornea and conjunctiva are able to absorb them [17].

\section{DRUG AND FORMULATION-RELATED FACTORS}

Drug activity, i.e. pharmacodynamics, is mediated by a pharmacophore, i.e. a group of atoms necessary to ensure supramolecular interactions with a specific target and trigger or block its biological response. Reaching the intended site by a drug, i.e. pharmacokinetics, is determined by its physicochemical properties, such as molecular size, lipophilicity/hydrophilicity, acidity, and charge. These properties can be manipulated during drug development to ensure optimal penetration through ocular barriers and effective exposure of a target. In some cases, the requirement of stability of a drug's active substance during its shelf-life may limit the available options of a drug formulation. For exam- 
ple, pilocarpine and epinephrine are stable only in acidic solutions, which on the other hand produce irritation immediately upon instillation and greatly increase lacrimation and drug dilution [18].

For ocular medicines applied as eye drops, enhancement of corneal permeability is favourably achieved by synthesising lipophilic ester prodrugs. When such prodrugs are topically applied, corneal epithelium and stroma act as a depot from which pharmacologically active acidic forms are liberated by corneal esterases. The prostaglandin analogues latanoprost and travoprost, currently the most frequently used anti-glaucoma drugs, are examples of such an approach [19]. Of note is that registration-wise, any new prodrug is a new drug that can be market-approved only on the basis of a full registration dossier.

The other way of enhancing corneal permeability is to add to a drug formulation an excipient that acts as a permeation enhancer, i.e. a substance that increases the corneal penetration of a drug by modifying the integrity of corneal epithelium. The permeation enhancer most frequently encountered in eye drops is benzalkonium chloride (BAK), which also acts as an antimicrobial preservative and a solubiliser of poorly water-soluble drugs. While effective in increasing the corneal permeability in vitro and in vivo of many drugs, upon prolonged exposure BAK displays several negative effects on the eye. The compound accumulates in ocular tissues, where it may remain for a long period of time and induce cell death in a dose-dependent manner. After topical drop instillation BAK has been detected in the trabecular meshwork (TM), corneal endothelium, lens, and retina $[20,21]$. The impact of prolonged BAK exposure has become a recognised issue in the management of glaucoma patients, particularly those with concurrent ocular surface disease. It has even been hypothesised recently that BAK may actually cause/worsen glaucoma, and this hypothesis is being tested experimentally in an animal model that closely reflects human physiology [22].

Preservatives, usually present in multi-use vials to prevent microbial contamination are blamed for conjunctival and palpebral side effects of eye drops [23], and sometimes also for damage of the ocular surface [24]. Preservative-free eye drops, packed in single-use containers or in bottles with aseptic filters, are better tolerated than drops containing preservatives, see e.g. [25]. Interestingly, among currently used preservatives BAK shows the highest promoting effect on transcorneal drug pen- etration [26], but its adverse effects are typically attributed to antimicrobial properties but not to permeability-enhancing properties. Moreover, as shown in a clinical trial, a preservative-free formulation of latanoprost displays non-inferior IOP-reducing activity compared to the reference BAK-containing latanoprost formula [27]. The relative importance of permeability enhancers for ocular pharmacokinetics may be much smaller than expected.

\section{DROP-RELATED FACTORS}

Besides the anatomical and physiological properties of the human eye and physicochemical properties of a drug substance and excipients, the delivery of a drug depends on the amount of drug solution instilled to the eye surface. This, in turn, depends on the physicochemical properties of a drug and its applicator, and on a technique used by a patient to instil it to the eye. The quantity of active substance applied in a single droplet depends on its concentration and the volume of the droplet, which then depends on fluid viscosity, surface tension, temperature, cohesion forces, adhesion to the dropper tip aperture, dimensions of the dropper tip, and application angle.

On the other hand, in the conjunctival sac only the predetermined amount of drug can be held. The cornea and conjunctiva of the eyeball and eyelids form a cul-de-sac with a volume of 7-9 $\mu \mathrm{L}$. By pulling the lower eyelid down this volume can be transiently increased to $30 \mu \mathrm{l}$, but reflective blinking reduces this effect and only $10 \mu \mathrm{l}$ of instilled solution will remain in contact with the eye for longer than a few seconds [15]. Any excess instilled solution is lost by overflow and may be drained by the lacrimal system, becoming available for systemic absorption. This may result in side effects, local and/or systemic. For example, overflow of eye drops containing a prostaglandin analogue results in lengthening, thickening, and hyperpigmentation of eyelashes, and hyperpigmentation of the skin around the eye [28].

Commercially available eyedroppers typically deliver $25.1 \mu \mathrm{L}$ to $56.4 \mu \mathrm{L}$, with an average drop volume of $39.0 \mu \mathrm{L}$ [29]. They are multi-dose or single-dose dispensers. In single-dose dispensers, which contain $0.1-1.0 \mathrm{~mL}$ of solution, the volume of the droplet depends on the dimensions of the orifice created in the tip. Difficulties in obtaining a smooth opening have been reported even when scissors were used. Multi-dose dispensers for eye drops usually consist of three parts: a bottle (volume between 2.5 and $15 \mathrm{~mL}$ ), a closure cap, and a tip (which 
is the most important element in determining the drop volume). The tip has a channel with duct with calibrated narrower inner and wider outer aperture, which ensures accurate dispensing of the solution and prevents jetting while squeezing the bottle. The drop size is proportionally dependent on the outer aperture diameter [30]. Decreasing the outer aperture size without changing the inner aperture diameter results in a respective decrease in drop weight, but the relationship between inner diameter and drop size is not linear [31]. Additionally, the outer orifice has a hemispherical surface where the drop is formed and expelled, which prevents from accidental injury of the eye during administration.

The most important physical factor influencing drop volume and its spherical shape is the surface tension of the fluid. According to Tate's law the weight of a drop is directly proportional to the surface tension of the solution. The volume of a droplet can be reduced by surface active substances (surfactants), which are added to eye drop formulations as preservatives and penetration enhancers. As mentioned previously, the most popular is benzalkonium chloride (BAK). Adding $0.01 \%$ of BAK to phosphate buffer solution reduces the droplet size from $43.7 \mu \mathrm{L}$ to $31.5 \mu \mathrm{L}$ [32]. When dispensing the smaller drops of the surface-active solutions, less force is needed to be exerted on the bottle and a lower dispensing time is noted. This could also be a practical advantage for elderly people experiencing physical difficulties with instilling their medication [33].

An important factor that determines the residence time of a drug on the eye surface is the viscosity of the instilled formulation. The viscosity of a fluid is a measure of its resistance to gradual deformation by shear stress or tensile stress; it is expressed in milipascal-seconds (mPa.s). Ocular contact time, and hence the bioavailability, of a drug can be enhanced by increasing the viscosity of the ophthalmic formulation. The addition of viscolysers, substances with high molecular weight, which barely cross biological membranes, prolongs the retention time of the instilled fluid [34]. Human tears have a viscosity of about $1.5 \mathrm{mPa}$.s. Retention of solutions starts to increase when the viscosity increases to $10 \mathrm{mPa} \cdot \mathrm{s}$ [35], but modulation of viscosity in the range 5 to $25 \mathrm{mPa}$.s barely influences the drop volume, which remains similar as in a non-viscous phosphate buffer solution. The ideal viscosity of ophthalmic solutions is estimated to be $15-30 \mathrm{mPa}$.s. Solutions with higher viscosity cause discomfort and damage to ocular epithelia due to an increase in the shear stresses during blinking, and interference with eyelid movements, vision, and patient comfort.

\section{PATIENT-RELATED FACTORS}

Patients' behaviours have a profound multifactorial influence on treatment performance. One factor is the angle of dispensing, the other is the number of drops reaching the ocular surface at a proper (or improper) time of day, and last but not least is longterm adherence to treatment, which is particularly important in the case of IOP-lowering anti-glaucoma therapy, which requires chronic application of eye drops, usually for the patient's lifetime.

Patient handling of ocular medicines and instillation technique influences drop size. It has been shown experimentally that the force used to squeeze the bottle dictates its volume. Squeezing the dropper bottle rapidly resulted in a higher weight of the drops dispensed and shorter time of their formation. Compression of the bottle with motor speed of $100 \mathrm{rpm}$ results in a $5 \%$ increase of drop volume compared with $30 \mathrm{rpm}$ [32]. This is a consequence of an increased tail at higher drop formation rates and extra impulse of liquid injected into the falling drop. At lower rates, the drop formation is slower and less or no extra liquid impulse is observed. By decreasing the speed of compressing the dropper bottle, the adsorption time is lengthened, resulting in a lower surface tension, which in turn influences the drop weight. It is recommended to instil eye drops with a $90^{\circ}$ angle, keeping the bottle above the eye. However, in practice this angle is usually much smaller, it can be as low as $30^{\circ}$. Changing the dispensing angle from $90^{\circ}$ to $45^{\circ}$ causes a decrease of the mean drop weight of the phosphate buffer solution by about $10 \%$. This can be explained by a reduction of the cross-sectional surface area of the outer aperture of the dropper tip [32].

When the strictest definition of noncompliance, i.e. not following the treatment as prescribed, was applied to a meta-analysis of data on treatment compliance, it transpired that up to $80 \%$ of glaucoma patients were non-compliant [36]. Some studies showed that less than one-third of the observed instillations were performed successfully. An objective evaluation of eye drop instillation in patients with glaucoma showed that the mean number of instilled drops per administration was $1.8-2.6[37,38]$, only up to $40 \%$ of fluid steam was instilled, and only $83 \%$ of patients were able to get the drop into the eye [39]. Interestingly, a recent four-year observa- 


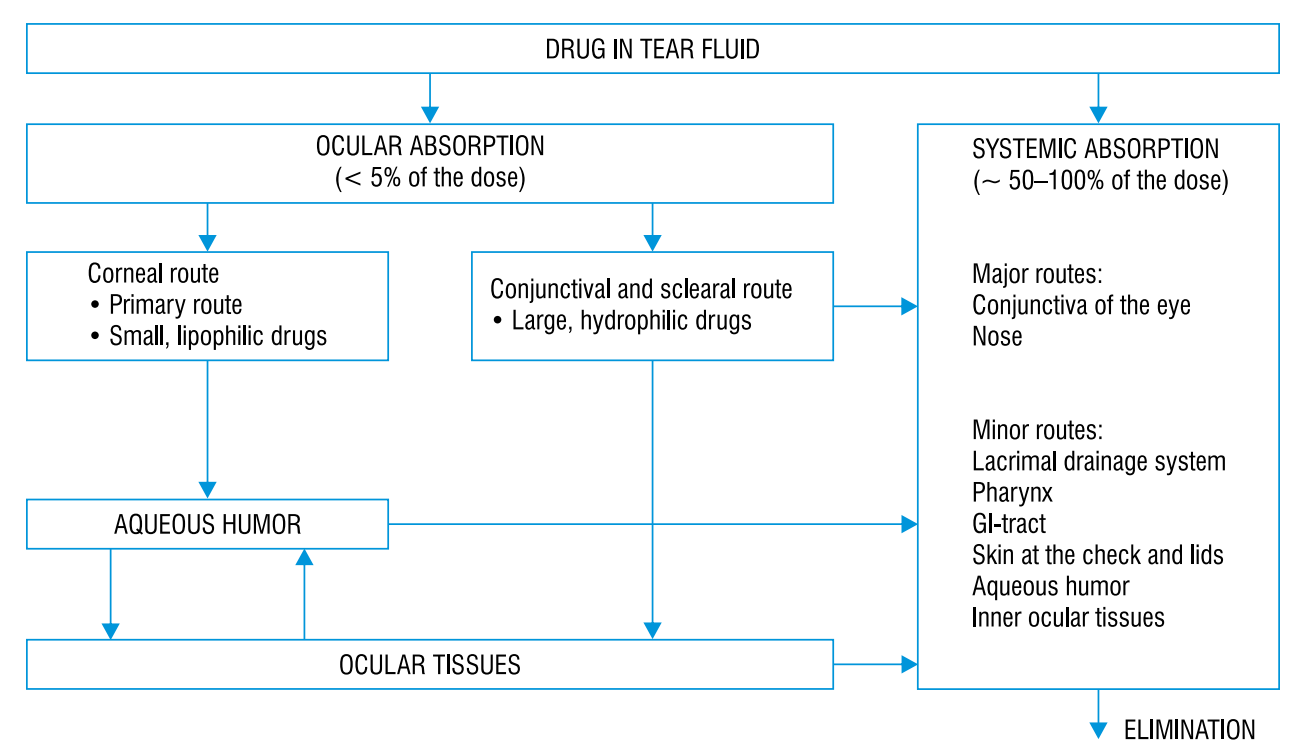

FIGURE 2. The fate of drugs instilled onto eye surface

tional study showed that adherence patterns observed in the first year of treatment reflect adherence patterns over the subsequent three years [40].

The issue of topical glaucoma therapy is complicated by the fact that the majority of primary open glaucoma patients are elderly. In a recent observational study [41] several barriers to optimal adherence were identified. The most frequently encountered were decreased self-efficacy, difficulty instilling drops, forgetfulness, and difficulties with the medication schedule.

\section{CONCLUSIONS}

Effective topical treatment of ocular diseases is a challenge. Even the best ocular drug will not be effective unless it is delivered to its site of action in sufficient quantity. Interplay of many factors influences the delivery of a medicine to its site of action. Some of these factors can be precisely shaped, for example concentration, structure, and properties of an active substance (or its prodrug) and excipients. These aspects are the subject of scientific studies on technologies to improve the bioavailability of drugs. Human factors that can influence the volume of the drug are more difficult to control, although they may drastically impair the effectiveness of treatment.

When a drug is instilled to the eye surface, the quantity that crosses the protective mechanisms of the eye is always much lower than that delivered in a drop formed by a dropper tip and applied into the conjunctival sac. It has been estimated that, even in optimal conditions, at most $5 \%$ of the instilled drug dose reaches the ocular target (Fig. 2). The volume exceeding the absorbed fraction does not implicate any therapeutic effect in the eye, thus we may conclude that even large differences in drop volume will have no influence on ocular drug performance.

There is only one exception to this rule. It concerns the possibility of non-ocular toxicity of an ocular drug. One such effect concerning overgrowth of eyelashes and hyperpigmentation of skin around the eye caused by the overspill of prostaglandin analogues has been mentioned previously. Even more important may be the possibility of peripherally-mediated effects of drugs applied topically to the eye, due to significant systemic absorption and bypassing liver first-pass metabolism. Bradycardia is a well-known effect of systemically absorbed fraction of ophthalmic timolol [42]. In the first seven years after market approval of ophthalmic timolol in the USA, 450 serious adverse cardiopulmonary events were reported, of which 32 were fatal [43].

As already mentioned, ophthalmic drugs are available for multi- or single-dose administration in a wide variety of glass or plastic dropper bottles that deliver drops with a volume between 25 and $70 \mu \mathrm{L}$. Almost 40 years ago it was proposed that a decrease in drop size would not only reduce the amount of overflow and the rate of drug loss through drainage, but also reduce the incidence of systemic side effects [44]. Since then it has been accepted that, from a biopharmaceutical point of view, volumes of 5 to $15 \mu \mathrm{L}$ should be instilled [45]. It is beyond 
the expertise of the authors of the present article to provide an explanation as to why the recommended smaller size of ocular drops is not common in commercially available eye drops.

\section{ACKNOWLEDGEMENTS}

This review article was supported by Polfa S.A., Warsaw, Poland.

\section{REFERENCES}

1. European Medicines Agency. Questions and answers on generic medicines. 2012, EMA/93905/2006 Rev. 2. http://www.ema. europa.eu/docs/en_GB/document_library/ Medicine_QA/2009/11/ WC500012382.pdf.

2. Cantor LB. Generic ophthalmic medications: as good as a Xerox? Medscape 2008. http://www.medscape.org/viewarticle/583866 print.

3. Mammo ZN, Flanagan JG, James DF, Trope GE. Generic versus brand-name North American topical glaucoma drops. Can J Ophthalmol 2012; 47: 55-61. doi:10.1016/j.jcjo.2011.12.004.

4. Leitritz MA, Lipp HP, Voykov B, Ziemssen F. Originalpräparat versus Generika - Latanoprost. Wie gleich ist unterschiedlich? Ophthalmologie 2015; 112: 127-139. doi: 10.1007/s00347-014-3097-x.

5. Cholkar K, Dasari SR, Pal D, Mitra AK. Eye: anatomy, physiology and barriers to drug delivery. In: Mitra AK (ed.). Ocular transporters and receptors: their role in drug delivery. Woodhead Publishing Series in Biomedicine 2012: 1-36.

6. Ruponen M, Urtti A. Undefined role of mucus as a barrier in ocular drug delivery. Eur J Pharm Biopharm 2015; 96: 442-446. doi:10.1016/j. ejpb.2015.02.032.

7. Kessing SV. Mucous gland system of the conjunctiva. A quantitative normal anatomical study. Acta Ophthalmol (Copenh) 1968 (Suppl. 95).

8. Watsky MA, Jablonski MM, Edelhauser HF. Comparison of conjunctival and corneal surface areas in rabbit and human. Curr Eye Res 1988; 7: 483-486.

9. Saha P, Kim KJ, Lee VH. A primary culture model of rabbit conjunctival epithelial cells exhibiting tight barrier properties. Curr Eye Res 1996; 15: 1163-1169. doi: 10.3109/02713689608995151.

10. Chang SC, Lee VH. Nasal and conjunctival contributions to the systemic absorption of topical timolol in the pigmented rabbit: implications in the design of strategies to maximize the ratio of ocular to systemic absorption. J Ocul Pharmacol 1987; 3: 159-169.

11. Geroski DH, Edelhauser HF. Transscleral drug delivery for posterior segment disease. Adv Drug Deliv Rev 2001; 52: 37-48. doi: 10.1016/ S0169-409X(01)00193-4.

12. Kim SH, Lutz RJ, Wang NS, Robinson MR. Transport barriers in transscleral drug delivery for retinal diseases. Ophthalmic Res 2007; 39: 244-254. doi: 10.1159/000108117.

13. Hornof $M$, Toropainen $E$, Urtti $A$. Cell culture models of the ocular barriers. Eur J Pharm Biopharm 2005; 60: 207-225.

14. Doane M. Blinking and the mechanics of the lacrimal drainage system. Ophthalmology 1981; 88: 844-851.

15. Mishima S, Gasset A, Klyce SD Jr., Baum JL. Determination of tear volume and tear flow. Invest Ophtalm 1966; 5: 264-276.

16. Shell JW. Pharmacokinetics of topically applied ophthalmic drugs. Surv Ophthalmol 1982; 26: 207-218.

17. Ooteghem MM. Factors influencing the retention of ophthalmic solutions on the eye surface. In: Saettone MS, Bucci G, Speiser P (eds.). Ophthalmic drug delivery. Vol. 11. Liviana Press, Padova: 7-17.

18. Achouri $D$, Alhanout $K$, Piccerelle $P$, Andrieu V. Recent advances in ocular drug delivery. Drug Dev Ind Pharm 2013; 39: 1599-1617. doi: 10.3109/03639045.2012.736515.

19. Peter A. Glaucoma Medical Therapy. 2nd. ed. Oxford University Press; New York 2008: 11-14.

20. Brignole-Baudouin $F$, Desbenoit N, Hamm Get al. A new safety concern for glaucoma treatment demonstrated by mass spectrometry imaging of benzalkonium chloride distribution in the eye, an experimental study in rabbits. PLoS One 2012; 7: e50180.
21. Desbenoit N, Schmitz-Afonso I, Baudouin C et al. Localisation and quantification of benzalkonium chloride in eye tissue by TOF-SIMS imaging and liquid chromatography mass spectrometry. Anal Bioanal Chem 2013; 405: 4039-4049.

22. Rasmussen CA, Kaufman PL, Kiland JA. Benzalkonium chloride and glaucoma. J Ocul Pharmacol Ther 2014; 30: 163-169.

23. Jaenen N, Baudouin C, Pouliquen P, Manni G, Figueiredo A, Zeyen T. Ocular symptoms and signs with preserved and preservative-free glaucoma medications. Eur J Ophthalmol 2007; 17: 341-349.

24. Mantelli F, Tranchina L, Lambiase A, Bonini S. Ocular surface damage by ophthalmic compounds. Curr Opin Allergy Clin Immunol 2011; 11:464-470.

25. Rosin LM, Bell NP. Preservative toxicity in glaucoma medication: clinical evaluation of benzalkonium chloride-free $0.5 \%$ timolol eye drops. Clin Ophthalmol 2013; 7: 2131-2135. doi: 10.2147/OPTH.S41358.

26. Kaur IP, Smitha R. Penetration enhancers and ocular bioadhesives: two new avenues for ophthalmic drug delivery. Drug Dev Ind Pharm 2002; 28: 353-369.

27. Sanford M. Preservative-free latanoprost eye drops in patients with primary open-angle glaucoma/ocular hypertension. Clin Drug Investig 2014; 34: 521-528. doi: 10.1007/s40261-014-0203-4.

28. Johnstone MA, Albert DM. Prostaglandin-induced hair growth. Surv Ophthalmol. 2002 Aug;47 Suppl 1:S185-202.

29. Lederer CM Jr, Harold RE. Drop size of commercial glaucoma medications. Am J Ophthalmol. 1986; 101: 691-694.

30. Brown J, Hotchkiss M, Davis B: Creating smaller eye drops by reducing eyedropper tip dimensions. Am J Ophthalmol 1985; 99: 460-464.

31. Brown R, Lynch M. Design of eyedropper tips for topical beta-blocking agents. Am J Ophthalmol 1986; 102: 123-124.

32. Van Santvliet L, Ludwig A. The influence of penetration enhancers on the volume instilled of eye drops. Eur J Pharm Biopharm 1998; 45: 189-198.

33. Van Santvliet L, Ludwig A. Influence of the physico-chemical properties of ophthalmic viscolysers on the weight of drops dispensed from a flexible dropper bottle. Eur J Pharm Sci 1999; 7: 339-345.

34. Koevary SB. Pharmacokinetics of topical ocular drug delivery. Curr. Drug Metab. 2003; 4: 213-222. doi: 10.2174/1389200033489488.

35. Zaki I, Fitzgerald P, Hardy JG, Wilson CG. A comparison of the effect of viscosity on the precorneal residence of solutions in rabbit and man. J Pharm Pharmacol 1986; 38: 463-466.

36. Olthoff CM, Schouten JS, van de Borne BW, Webers CA. Noncompliance with ocular hypotensive treatment in patients with glaucoma or ocular hypertension an evidence-based review. Ophthalmology. 2005; 112: 953-961.

37. Kass MA, Hodapp E, Gordon M, Kolker AE, Goldberg I. Patient administration of eyedrops, part I: interview. Ann Ophthalmol 1982; 14: 775-779.

38. Kass MA, Hodapp E, Gordon M, Kolker AE, Goldberg I. Patient administration of eyedrops, part II: observation. Ann Ophthalmol 1982; 14: 889-893.

39. Brown MM, Brown GC, Spaeth GL. Improper topical self-administration of ocular medication among patients with glaucoma. Can J Ophthalmol 1984; 19: 2-5.

40. Newman-Casey PA, Blachley T, Lee PP, Heisler M, Farris B, Stein JD. Patterns of glaucoma medication adherence over four years of follow-up. Ophthalmology 2015; 122: 2010-2021. doi:10.1016/j. ophtha.2015.06.039.

41. Newman-Casey PA, Robin AL, Blachley T et al. The most common barriers to glaucoma medication adherence: A cross-sectional survey. Ophthalmology 2015; 122:1308-1316. doi:10.1016/j.ophtha.2015.03.026.

42. Pratt NL, Ramsay EN, Kalisch Ellett LM, Nguyen TA, Roughead EE. Association between ophthalmic timolol and hospitalisation for bradycardia. J Ophthalmol 2015; 567387. doi: 10.1155/2015/567387.

43. Nelson WL, Fraunfelder FT, Sills JM, Arrowsmith JB, Kuritsky JN. Adverse respiratory and cardiovascular events attributed to timolol ophthalmic solution, 1978-1985. Am J Ophthalmol 1986; 102: 606-611.

44. Patton TF. Pharmacokinetic evidence for improved ophthalmic drug delivery by reduction of instilled volume. J Pharm Sci. 1977; 66: 1058-1059.

45. Van Santvliet L, Ludwig A. Determinants of eye drop size. Surv Ophthalmol 2004; 49: 197-213. 